\title{
Aberrant Left Brachiocephalic Vein versus Persistent Left Superior Vena Cava without Bridging Vein in Adults: Evaluation on Computed Tomography
}

\author{
Makiko Kobayashi, MD, ${ }^{1,2}$ Tamaki Ichikawa, MD, PhD, ${ }^{1}$ Jun Koizumi, MD, PhD, ${ }^{1}$ \\ Jun Hashimoto, MD, PhD, ${ }^{1}$ Hiroshi Yamamuro, MD, PhD, ${ }^{1}$ Takuya Hara, MD, ${ }^{1}$ \\ Takakiyo Nomura, MD, ${ }^{1}$ Shigetaka Kanda, MD, PhD, ${ }^{3}$ and Yutaka Imai, MD, PhD ${ }^{1}$
}

Objective: Persistent left superior vena cava without bringing vein (PLSVC $\mathrm{w} / \mathrm{o} \mathrm{BV}$ ) is a common thoracic venous anomaly, while aberrant left brachiocephalic vein (ALBCV) is an uncommon condition. We compared the incidences of the two venous anomalies and assessed congenital cardiovascular diseases (CCDs) in adults using computed tomography $(\mathrm{CT})$.

Materials and Methods: We reviewed the recorded reports or CT images of 49,494 adults for PLSVC w/o BV and ALBCV in two hospitals. We determined incidences of two venous anomalies and the rate of associated CCDs.

Results: 76 PLSVCs w/o BV and 27 ALBCVs were found. The incidence of PLSVC $\mathrm{w} / \mathrm{o}$ BV was $0.15 \%$ and the incidence of ALBCV was $0.055 \%$. PLSVC w/o BV had higher incidence than ALBCV $(p<0.001)$. Four PLSVCs w/o BV and one ALBCV were associated with congenital heart diseases. Two PLSVCs w/o BV and four ALBCVs were associated with congenital aortic arch anomaly (CAAA). ALBCV had higher incidence of associated CAAA than PLSVC $w / o$ BV $(P=0.02)$. Conclusion: The incidence of ALBCV was $<50 \%$ that of PLSVC w/o BV. The two venous anomalies found on CT during adulthood were rarely associated with CCDs.

${ }^{1}$ Department of Radiology, Tokai University School of Medicine, Isehara, Kanagawa, Japan

${ }^{2}$ Division of Diagnostic Radiology, Hiratsuka City Hospital, Hiratsuka, Kanagawa, Japan

${ }^{3}$ Department of Cardiac Medicine, Tokai University School of Medicine, Isehara, Kanagawa, Japan

Received: September 2, 2018; Accepted: September 29, 2018 Corresponding author: Tamaki Ichikawa, MD, PhD. Department of Radiology, Tokai University School of Medicine, 143 Shimokasuya, Isehara, Kanagawa 259-1193, Japan

Tel: +81-463-93-1121, Fax: +81-463-03-6827

E-mail: tamaki-i@is.icc.u.tokai.ac.jp

(cc) BY-NC-SA (C2018 The Editorial Committee of Annals of Vascular Diseases. This article is distributed under the terms of the Creative Commons Attribution License, which permits use, distribution, and reproduction in any medium, provided the credit of the original work, a link to the license, and indication of any change are properly given, and the original work is not used for commercial purposes. Remixed or transformed contributions must be distributed under the same license as the original.
Keywords: persistent left superior vena cava, aberrant left brachiocephalic vein, computed tomography, transverse venous plexus

\section{Introduction}

Persistent left superior vena cava (PLSVC) is the most common venous anomaly of the thorax and occurs in approximately $0.3 \%-0.5 \%$ of the general population and in $<10 \%$ of patients with congenital heart disease (CHD). ${ }^{1-5)}$ There are three types of PLSVC; double superior vena cava (SVC) with and without bridging vein, and PLSVC with absent right SVC. ${ }^{1-5)}$ More than $90 \%$ of PLSVCs are double SVCs, and PLSVC without bridging vein (w/o BV) accounts for $60 \%-70 \%$ of double SVCs. ${ }^{2,4)}$ PLSVC is formed from remaining right and left anterior cardinal veins, and PLSVC w/o BV results from regression of both the superior and inferior transverse venous plexuses around the primitive aorta (Fig. 1). ${ }^{1-7)}$

In the first 6 weeks of gestation, the superior and inferior transverse venous plexuses present around the primitive aorta. Then the 8 th week of gestation, the inferior transverse venous plexus (TVP) regresses and the superior TVP remains, which finally forms a normal left brachiocephalic vein (BCV) (Fig. 1) ${ }^{8}{ }^{8}$ When the superior TVP regresses and the inferior TVP remains, an aberrant left brachiocephalic vein (ALBCV) develops, and most are retroaortic courses. ${ }^{8-15)}$ ALBCV is rare and its incidence in child patients with $\mathrm{CHD}$ has been reported to be $0.98 \%-$ $1.7 \%{ }^{8,12)}$ The incidence of ALBCV was higher in patients with conotruncal and congenital aortic arch anomaly (CAAA). ${ }^{8,10,11,13-15)}$ There was no report of the incidence on adults including patients with CHD.

Since PLSVCs w/o BV and ALBCVs tend to be associated with congenital cardiovascular diseases (CCDs) including CHD and CAAA, in childhood associated CCDs are problem.

The isolated venous anomalies tend to be regarded sim- 
a

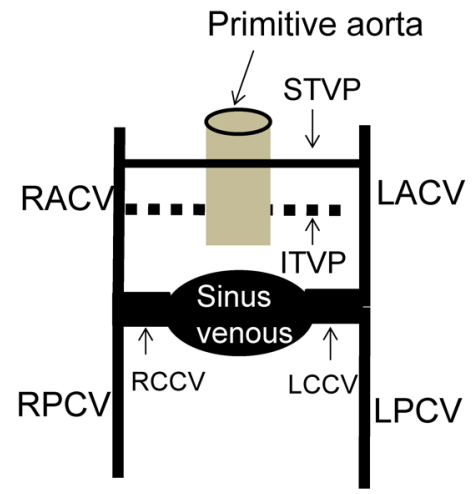

Persistent LSVC without bridging vein

C

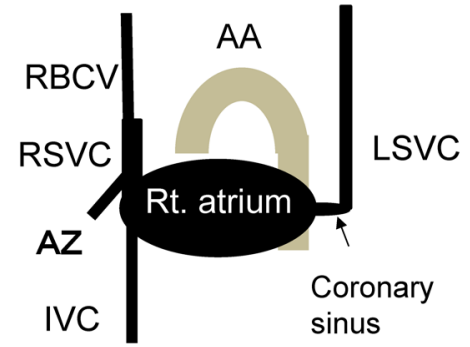

b

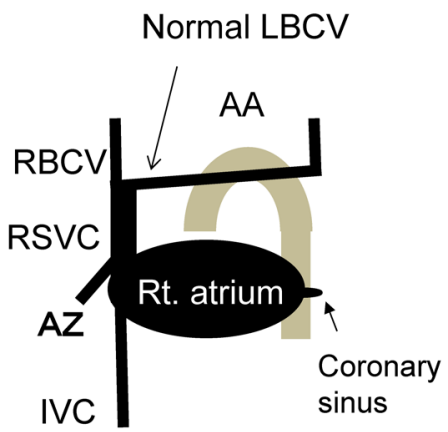

d

Aberrant LBCV

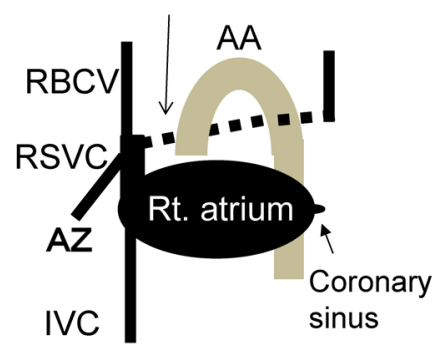

Fig. 1 Schematic diagrams of venous development. a. Venous channels arise from 3 major cardinal veins (the anterior, posterior, and common cardinal veins). Superior and inferior transverse venous plexuses are present around the primitive aorta at the sixth week of gestation. b. Normal development of thoracic veins. Both anterior cardinal veins interconnect with the superior transverse venous plexus. The aortic arch occupies the embryonal space of the inferior transverse venous plexus and a normal left brachiocephalic vein passes anterior to the aortic arch. c. The persistent left superior vena cava occurs because the left anterior cardinal vein remains and there is regression of both the superior and inferior transverse venous plexuses. $\mathbf{d}$. The aberrant left brachiocephalic vein occurs because the inferior transverse venous plexus remains and there is regression of the superior transverse venous plexus. RACV: right anterior cardinal vein; LACV: left anterior cardinal vein; STVP: superior transverse venous plexus; ITVP: inferior transverse venous plexus; RPCV: right posterior cardinal vein; LPCV: left posterior cardinal vein; RCCV: right common cardinal vein; LCCV: left common cardinal vein; RBCV: right brachiocephalic vein; LBCV: left brachiocephalic vein; RSVC: right superior vena cava; LSVC: left superior vena cava; AA: aortic arch; AZ: azygos vein; IVC: inferior vena cava

ply as normal variants. However, identification of major thoracic venous anomalies on radiologic examination is important for placing central venous line (CVL) or intracardiac electrode/pacemaker. ${ }^{2,4,8,12)}$

The incidence of ALBCV found in adulthood was not as rare as described in the previous literature. ${ }^{14)}$ To the best of our knowledge, there are no reports that compare the incidences of ALBCV and PLSVC w/o BV. Clinical significance of the two venous anomalies in adulthood remains unclear. So, we determined the incidences of two venous anomalies and evaluated associated CCDs in adults on computed tomography (CT).

\section{Materials and Methods}

This retrospective study was approved by our institutional review board (16R-253).

Our study included 49,494 adults (27,282 males and 22,212 females; mean age: $62 \pm 19.1$ years) who underwent CT examinations in two university hospitals from January 2013 to July 2018. One was Tokai University Hospital and the other was Tokai University Tokyo Hospital.

In Tokai University Hospital, 42,791 chest CT examinations were performed during the study period. Among these reports made by board-certified radiologists with more than six years of experience each, the patients with ALBCV and PLSVC w/o BV were searched by using the 
key words "double SVC," "persistent left SVC," "absent SVC," "aberrant or absent BCV" or "innominate vein," "pulmonary venous return," "vena cava anomalies," and “absent right SVC."

In Tokai University Tokyo Hospital, 6,703 chest CT examinations were performed during the study period. Two board-certified radiologists with more than 15 years of experience each (TI, JK) reviewed CT images to search for patients with PLSVC w/o BV and ALBCV.

For PLSVC w/o BV, we assessed the draining point of PLSVC. For ALBCV, we assessed the abnormal position of the ALBCV and the number of left BCV. CCDs were assessed using the CT images and medical records in both groups of PLSVC w/o BV and ALBCV. We considered the aortic arch which was seen above the lower margin of the clavicle to be a high aortic arch. ${ }^{11)}$ Venous intervention such as CVL insertion and pacemaker placement were assessed.

Patients in Tokai University Hospital were scanned using a 64 to 128-slice scanner (Somatom Sensation Cardiac 64, Definition, Definition Flash, Definition Edge, Definition AS + ; Siemens AG, Munich, Germany). Patients in Tokai University Tokyo Hospital were scanned using a 64 slice scanner (Aquilion, Canon, Otawara, Japan). The scanning parameters and CT protocol were the same in both hospitals. The detailed scanning parameters were as follows: slice thickness of $0.5 \mathrm{~mm}$, tube voltage, $120 \mathrm{kVp}$; tube current, auto $\mathrm{mA}$; and rotation time, $0.5 \mathrm{~s}$. Contrastenhanced CT examinations were performed by injecting $2 \mathrm{~mL} / \mathrm{kg}$ of nonionic contrast material $(300 \mathrm{mgI} / \mathrm{mL})$ at a rate of $2 \mathrm{~mL} / \mathrm{s}$ with a 120 -s scanning delay. CT angiography was performed using the bolus tracking method and an injection of $100 \mathrm{~mL}$ of nonionic contrast material $(350-370 \mathrm{mgI} / \mathrm{mL})$ at a rate of $4 \mathrm{~mL} / \mathrm{s}$. Coronary CT angiography was performed using test injection method by injecting $40-70 \mathrm{~mL}$ of nonionic contrast material $(300 \mathrm{mgI} / \mathrm{mL})$ at a rate of $3.5-4.5 \mathrm{~mL} / \mathrm{s}$.

Two board-certified radiologists with more than 15 years of experience (TI, JK) interpreted CT images of the two venous anomalies using the $1-5-\mathrm{mm}$ reconstructed axial CT images on a picture archiving and communication system workstation. Additional multiplanar reformations and three dimensional images were used for the assessment when needed. They resolved any disagreement through discussion to reach consensus.

We compared the incidences of the venous anomalies and associated CCD using Fisher's exact test. P values less than 0.05 were defined to indicate statistical significance. Statistical analysis was performed using SPSS version 23 software (IBM Co., Armonk, NY, USA).

\section{Results}

A total of 49,494 adults $(27,282$ males and 22,221 females; mean age: $62.5 \pm 16.2$ years) underwent chest CT examinations during the study period (Tokai University Hospital: 23,880 males and 18,911 females; mean age: $64.6 \pm 16.8$ years, Tokai University Tokyo Hospital: 3,402 males and 3,301 females; mean age: $58 \pm 16$ years). Numbers and incidences of venous anomalies in two hospitals were shown in Table 1.

Seventy-six PLSVC w/o BV patients (45 males and 31 females; mean age: $60 \pm 18$ years) and 27 ALBCV patients (16 males and 11 females; mean age: $61 \pm 15$ years) were found. In the PLSVC w/o BV group, 56 patients could be evaluated on contrast-enhanced CT, including 20 CT angiographies. In the ALBCV group, 21 patients could be evaluated on contrast-enhanced CT including eight CT angiographies.

The incidence of PLSVC w/o BV was $0.15 \%$ and the incidence of ALBCV was $0.055 \%$. The incidences of PLSVC w/o BV were higher than those of ALBCV $(\mathrm{P}=0.001)$.

Four patients with PLSVC w/o BV and one patient with ALBCV had CHDs (Table 2). The incidences of associated CHD were $5.3 \%$ in the PLSVC w/o BV group and 3.8\% in the ALBCV group. There were no significant differences in the incidences of associated CHD between two groups $(\mathrm{P}=0.62)$. Two patients with Tetralogy of Fallot (TOF) were associated with an ALBCV with right aortic arch with mirror image branching and a PLSVC w/o BV with polysplenia (No. 1 and 3 in Table 2). Five patients with ventricular septal defect (VSD) including the two patients with TOF had reserved operations (Table 2). One patient with ALBCV had CHD and CAAA (No. 1 in Table 2).

Two patients with PLSVC w/o BV and four patients with ALBCV had CAAA (Fig. 2). Two adults of PLSVC w/o BV were associated with incomplete double aortic arches and four adults of ALBCV were associated with

Table 1 Numbers and incidences of venous anomalies in two hospitals

\begin{tabular}{lrr}
\multicolumn{1}{c}{ Patient numbers of two hospitals } & Number and & Incidence \\
& PLSVC w/o BV & ALBCV \\
\hline Tokai University Hospital $(42,791)$ & $63(0.15 \%)$ & $21(0.049 \%)$ \\
Tokai University Tokyo Hospital $(6,703)$ & $13(0.19 \%)$ & $6(0.089 \%)$ \\
Total $(49,494)$ & $76(0.15 \%)$ & $27(0.055 \%)$ \\
\hline
\end{tabular}

ALBCV: aberrant left brachiocephalic vein; PLSVC w/o BV: persistent left superior vena cava without bridging vein 
Table 2 Associated congenital heart disease and others

\begin{tabular}{ccccl}
\hline No & Venous anomaly & Age & Sex & \\
\hline 1 & ALBCV & 49 & M & TOF (PS, VSD), patent foramen ovale, RAMI \\
2 & PLSVC w/o BV & 37 & M & VSD \\
3 & PLSVC w/o BV & 34 & M & TOF (PS, VSD), Absent PV \\
& & & Polysplenia, absent IVC with AZ continuation \\
4 & PLSVC w/o BV & 27 & F & Double outlet right ventricle (VSD), left IVC \\
& PLSVC w/o BV & 51 & M & Anomalous origin of left circumflex artery
\end{tabular}

ALBCV: aberrant left brachiocephalic vein; PLSVC w/o BV: persistent left superior vena cava without bridging vein; TOF: Tetralogy of Fallot; PS: pulmonary stenosis; VSD: ventricular septal defect; RAMI: right aortic arch with mirror image branching; PV: portal vein; IVC: inferior vena cava; AZ: azygos vein; M: male; F: female

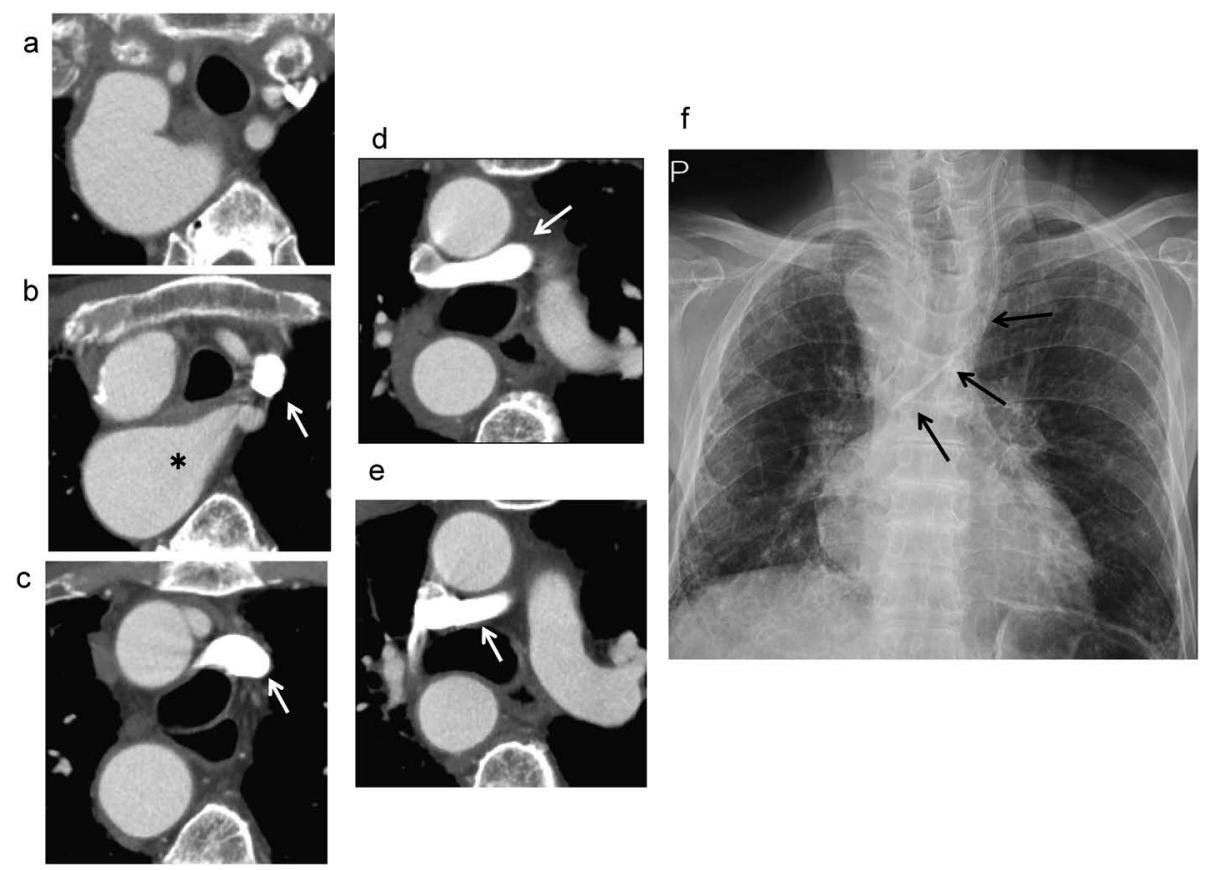

Fig. 2 A case of aberrant left brachiocephalic vein (ALBCV) coexisting with right aortic arch with aberrant left subclavian artery. Axial computed tomography images (a-e) show the right aortic arch with aberrant left subclavian artery and highly enhanced retroaortic left brachiocephalic vein by left hand injection of contrast material (white arrows). The asterisk indicates the Kommerell diverticulum (b). Chest radiograph (f) shows central venous line through the ALBCV (black arrows). The right aortic arch appears in the high position.

CAAA, including an incomplete double aortic arch, a right aortic arch with mirror image branching, a right aortic arch with aberrant left subclavian artery, and a coarctation. The incidence of associated CAAA was $2.7 \%$ in the PLSVC w/o BV group and $15 \%$ in the ALBCV group. The ALBCV group had higher incidence of associated CAAA than the PLSVC w/o BV group $(\mathrm{P}=0.02)$.

Fourteen patients with ALBCV had high aortic arches and two patients of PLSVC w/o BV were associated with high level incomplete double aortic arch.

All patients with CHD and CAAA underwent transthoracic echocardiographic examinations.
One patient with Marfan syndrome was found in the PLSVC w/o BV. She had no CHD but had a right tracheal bronchus, left bronchial diverticulum, scoliosis, and lens subluxation.

Seventy-four PLSVCs w/o BV drained into the right atrium via the coronary sinus. On two patients with PLSVC w/o BV including a patient of double outlet right ventricle, PLSVCs drained into the azygos vein via the hemiazygos vein, and they were associated with the left inferior vena cava (No. 4 in Table 2). In three patients with PLSVC w/o $\mathrm{BV}$, pacemaker insertion from the left SVC was not performed (Fig. 3). One of the patients underwent myocar- 


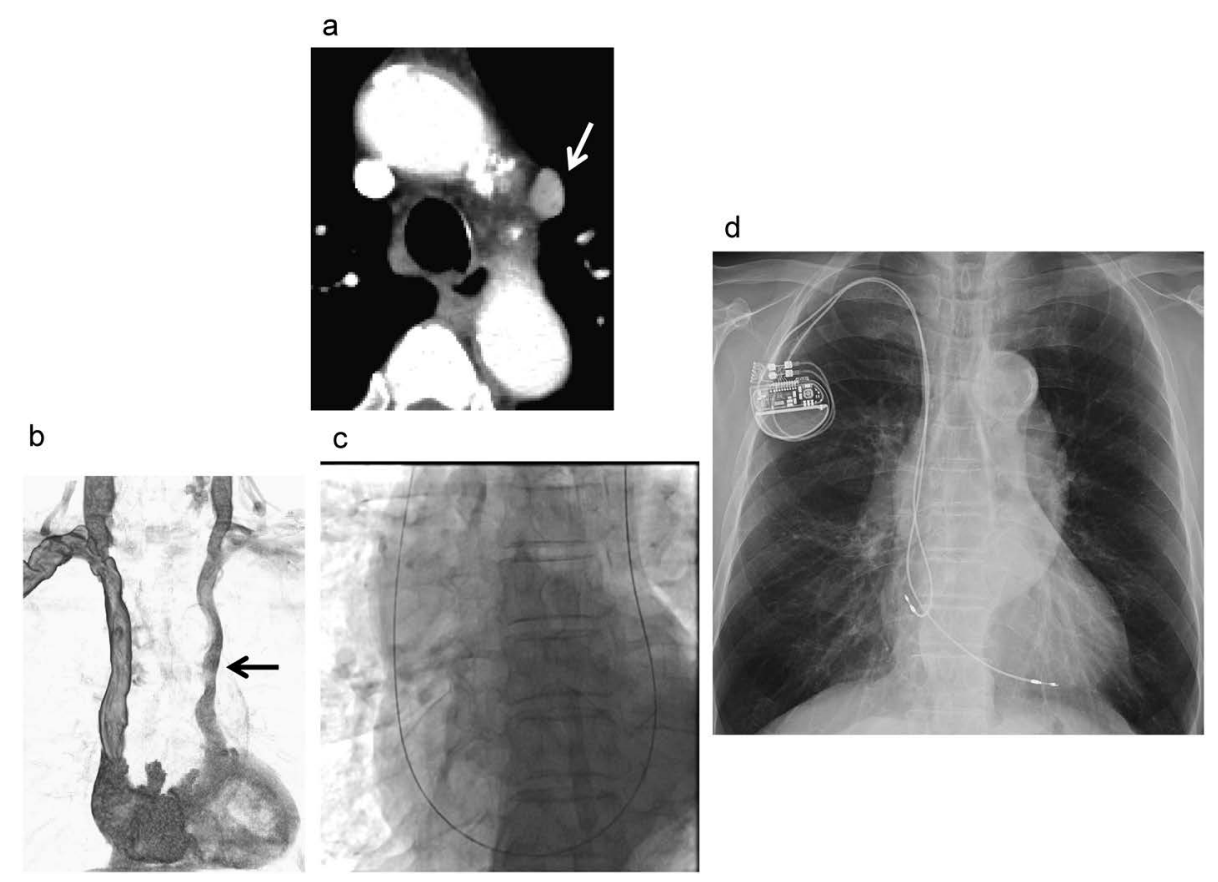

Fig. 3 A patient with a persistent left superior vena cava (PLSVC) who could not undergo pacemaker implantation from the left superior vena cava (SVC). Axial computed tomography (a) and volume rendering (b) images show the PLSVC (arrows). Angiographic image (c) shows a guidewire inserted from the left subclavian vein draining to the right SVC via the left SVC. Plain radiograph shows pacemaker implantation from the right SVC (d).

dial electrode implantation by the subxiphoid approach. In two patients with the PLSVC w/o BV, central venous catheters were inserted from the left jugular vein into the PLSVC due to malignant lymphoma.

Twenty-six patients with ALBCV had retroaortic left BCVs. In one patient with supraaortic left BCV, an ALBCV coursed between the right brachiocephalic and left common carotid arteries and was associated with PLSVC. Two patients had double left BCVs including normal position and retroaortic left BCVs. Two patients with ALBCV underwent central venous catheter insertions safely (Fig. 2).

\section{Discussion}

In this study, the incidence of PLSVCs w/o BV in adults determined by CT evaluation was $0.15 \%$, which was similar to the incidence in general population on previous reports. ${ }^{2,3)}$

A high incidence of CHD associated with PLSVC has been determined by angiography or echocardiography in children. ${ }^{3,16,17)}$ Steckiewicz et al. reported that eight adults with PLSVC were found in 4,708 cardiac implantable electronic device recipients and no one was associated with CHD. ${ }^{18)}$ In our study, only four adults with PLSVC w/o BV had CHD. The most common associated CHD is VSD, and others include TOF and pulmonary stenosis. $3,6,7,16)$ In our study, five VSD cases, including two TOF cases, were found. However, PLSVCs detected during adulthood on less-invasive radiological examinations such as CT or MRI are typically asymptomatic. PLSVC without CHD in adults is diagnosed incidentally during CVL placement, and pacemaker and implantable cardioverter defibrillator implantation, and CT examinations. ${ }^{2,6,18)}$

ALBCV is very rare, and Chen et al. reported that the incidence of ALBCV in 1,812 patients with CHD was approximately $1.7 \% .{ }^{8)}$ Kahkouee et al. reported that among 22 patients with ALBCV with a mean age of 12.5 years, only one patient had isolated ALBCV $(4.5 \%) .{ }^{15)}$ Similar to PLSVC, ALBCV found during childhood has a tendency to be associated with CHD. ${ }^{8,12,15,17-19)} \mathrm{Kim}$ et al. reported that among 14 patients found in adulthood, only one ALBCV was associated with $\mathrm{CHD}$, and this result was similar to ours. ${ }^{11)}$ In Tokyo Hospital, 19 venous anomalies were not associated with CHD because of small subject size. Yamamuro et al. reported that the incidence of ALBCV in adults without CHD found on CT was $0.03 \%-0.062 \% .{ }^{14)}$ In our study, the incidence of ALBCV in adults was $0.055 \%$. The incidence of ALBCV was similar to previous reports and were also not associated with CHD. ${ }^{14)}$

Our study was the first one to compare the incidences of PLSVC w/o BV and ALBCV in adults using CT, and 
results indicate that the incidence of ALBCV was less than half of PLSVC w/o BV. Since PLSVC without right SVC accounted for about $10 \%$ of all PLSVC cases, ${ }^{20,21)}$ our study indicates that the ALBCV had higher incidence than PLSVC without right SVC.

Simple failure of obliteration of the left anterior cardinal vein results in PLSVC. 1,2,6) Failing to obliterate the ligament of Marshall leads to the formation of a left SVC that drains into the coronary sinus. $\left.{ }^{6}\right)$ When the superior and inferior TVP around the primitive aorta regress, the PLSVC w/o BV is formed (Fig. 1). The embryologic cause of PLSVC is unknown despite the tendency to be associated with CHD.

ALBCV is related to the development of the height of the aortic arch and the prevention of normal shortening of aortic arch during embryogenesis. ${ }^{8-11)}$ In our study, 12 CAAAs, including high aortic arch, were found in 27 cases of ALBCV. The high aortic arch might be formed from the third pharyngeal arch artery, which differs from the normal aortic arch formed from the fourth pharyngeal arch artery. ALBCV patients had higher incidence of having CAAA than PLSVC w/o BV patients. However, the exact embryogenesis of ALBCV remains unknown. ${ }^{8,14}$

PLSVC is not associated with a retroaortic left BCV because the proximal part of the retroaortic left $\mathrm{BCV}$, which is a part of the inferior TVP, derives from the distal portion of the left common cardinal vein. ${ }^{8)}$ In our study, an ALBCV was associated with PLSVC because the supraaortic left $\mathrm{BCV}$ formed from the remaining anomalous superior TVP.

In previous studies, most PLSVCs drain into the right atrium via the coronary sinus as our cases did. ${ }^{1-3,5,7)}$ On rare occasions, the coronary sinus is absent, and the PLSVC drains directly into the right atrium. ${ }^{6,7)}$ Additionally, the PLSVC can connect directly or through the pulmonary veins to the left atrium, which causes a right to left shunt. ${ }^{6,7)}$

Because an ALBCV that develops from the lower anastomosis is joined by the right $\mathrm{BCV}$ to the SVC, the SVC segment has to be shorter than normal, and the azygos vein has to be situated at the same level or higher than the root of the SVC. ${ }^{13)}$

An isolated PLSVC or ALBCV is a normal variant, and does not have any clinical significance. However, the exact anatomical diagnoses of PLSVC and ALBCV are important prior to interventional procedures. The technical difficulties associated with PLSVC may lead to the misplacement of a catheter and the vessel wall injury by the catheter. ${ }^{2,6)}$ Serious complications, including angina, arrhythmia, cardiogenic shock, and even cardiac arrest have been reported when a guide wire or catheter is manipulated via the PLSVC. ${ }^{2,6)}$ In our study, to avoid complications due to PLSVC, cardiac pacemaker placements from right subclavian vein through the right SVC were performed in three patients, and a myocardial electrode implantation was performed by the subxiphoid approach in one patient. Meanwhile, two central catheter insertions and one cardiac pacemaker placement through the ALBCV were performed on patients with ALBCV carefully because the clinicians were informed of the anomalies by CT reports previously (Fig. 2). If incorrect insertion into the azygos vein at the same confluence level of the ALBCV can be prevented, $\mathrm{CV}$ catheter placement through ALBCV might be safe because download via the ALBCV is more gentle than that via the normal supraaortic left BCV.

Left partial anomalous pulmonary venous return (PAPVR) must be considered differential diagnosis of the PLSVC and ALBCV. ${ }^{2,14,19,20)}$ Because PAPVR was found to occur in $0.04 \%$ of the population in a post-mortem series, more often on the right than on the left, the incidence of ALBCV may be higher than that of left PAPVR, which is frequently detected on CT. ${ }^{14,22-24)}$

Our study had some limitations. At first, search methods were different in two hospitals: one was key word search method and the other was direct CT image review method. When we used a keyword search of CT reports in Tokai University Hospital, in some cases, the desired venous anomalies might not have been detected. However, the incidences of both venous anomalies were similar to those of previous reports. ${ }^{1-3,14)}$ Second, because not all patients underwent echocardiography, minor CHD, such as a tiny atrial septal defect, could not be detected. Third, not all patients underwent cardiac CT and therefore, we could not evaluate unroofed coronary sinus and coronary sinus atresia, so both coronary diseases coexisted with PLSVC. ${ }^{25-27)}$ Finally, the number of patients examined by CT was too small to evaluate associated diseases and a larger study using the direct image review method is necessary to evaluate the incidence, exact anatomy of both venous anomalies and associated complications.

\section{Conclusion}

We assessed CT images of PLSVC w/o BV and ALBCV in adults. The incidence of ALBCV was less than half that of PLSVC w/o BV. The two venous anomalies were rarely associated with CHD and CAAA. Identification of those anomalies on CT, even if isolated venous anomalies, are important for correct planning of CVL and cardiac pacemaker placement.

\section{Acknowledgments}

We thank Kenji Kasahara, Yuuki Sato and Fumie Samatsu for technical assistance. 


\section{Disclosure Statement}

There are no conflicts of interest to declare.

\section{Author Contributions}

Study conception: MK, TI

Data collection: MK, TI

Analysis: MK

Investigation: MK, TI, JK

Writing: MK

Funding acquisition: JK

Critical review and revision: all authors

Final approval of the article: all authors

Accountability for all aspects of the work: all authors

\section{References}

1) Minniti S, Visentini S, Procacci C. Congenital anomalies of the venae cavae: embryological origin, imaging features and report of three new variants. Eur Radiol 2002; 12: 2040-55.

2) Ratliff HL, Yousufuddin M, Lieving WR, et al. Persistent left superior vena cava: case reports and clinical implications. Int J Cardiol 2006; 113: 242-6.

3) Kula S, Cevik A, Sanli C, et al. Persistent left superior vena cava: experience of a tertiary health-care center. Pediatr Int 2011; 53: 1066-9.

4) Biffi M, Bertini M, Ziacchi M, et al. Clinical implications of left superior vena cava persistence in candidates for pacemaker or cardioverter-defibrillator implantation. Heart Vessels 2009; 24: 142-6.

5) Kurtoglu E, Cakin O, Akcay S, et al. Persistent left superior vena cava draining into the coronary sinus: a case report. Cardiol Res 2011; 2: 249-52.

6) Bhatti S, Hakeem A, Ahmad U, et al. Persistent left superior vena cava (PLSVC) with anomalous left hepatic vein drainage into the right atrium: role of imaging and clinical relevance. Vasc Med 2007; 12: 319-24.

7) Peltier J, Destrieux C, Desme J, et al. The persistent left superior vena cava: anatomical study, pathogenesis and clinical considerations. Surg Radiol Anat 2006; 28: 206-10.

8) Chen SJ, Lui KL, Chen HY, et al. Anomalous brachiocephalic vein: CT, embryology, and clinical implications. Am J Roentgenol 2005; 184: 1235-40.

9) Takada Y, Narimatsu A, Kohno A, et al. Anomalous left brachiocephalic vein: CT findings. J Comput Assist Tomogr 1992; 16: 893-6.

10) Minami M, Noda M, Kawauchi N, et al. Postaortic left innominate vein: radiological assessment and pathogenesis. Clin Radiol 1993; 48: 52-6.

11) Kim SH, Chung JW, Im JG, et al. Subaortic left innominate vein: radiologic findings and consideration of embryogenesis. J Thorac Imaging 1999; 14: 142-6.

12) Choi JY, Jung MJ, Kim YH, et al. Anomalous subaortic position of the brachiocephalic vein (innominate vein): an echocardiographic study. Br Heart J 1990; 64: 385-7.

13) Chern MS, Ko JSC, Tsai A, et al. Aberrant left brachiocephalic vein: CT imaging findings and embryologic correlation. Eur Radiol 1999; 9: 1835-9.

14) Yamamuro H, Ichikawa $T$, Hashimoto J, et al. Congenital anomalies of the left brachiocephalic vein detected in adults on computed tomography. Jpn J Radiol 2017; 35: 597-605.

15) Kahkouee S, Sadr M, Pedarzadeh E, et al. Anomalous left brachiocephalic vein: important vascular anomaly concomitant with congenital anomalies and heart diseases. Folia Morphol (Warsz) 2017; 76: 51-7.

16) Ari ME, Doğan V, Özgür S, et al. Persistent left superior vena cava accompanying congenital heart disease in children: experience of a tertiary care center. Echocardiography 2017; 34: 436-40.

17) Postema PG, Rammeloo LA, van Litsenburg R, et al. Left superior vena cava in pediatric cardiology associated with extra-cardiac anomalies. Int J Cardiol 2008; 123: 302-6.

18) Steckiewicz R, Świętoń EB, Czerniawska J, et al. Imaging topography and morphometry of persistent left superior caval vein and its variations, detected on cardiac implantable electronic device implantation. Folia Morphol (Warsz) 2017; 76: 58-65.

19) Curtil A, Tronc F, Champsaur G, et al. The left retro-aortic brachiocephalic vein: morphologic data and diagnostic ultrasound in 27 cases. Surg Radiol Anat 1999; 21 S4: 251-4.

20) Uemura M, Suwa F, Takemura A, et al. Classification of persistent left superior vena cava considering presence and development of both superior venae cavae, the anastomotic ramus between superior venae cavae, and the azygos venous system. Anat Sci Int 2012; 87: 212-22.

21) Bartram U, Van Praagh S, Levine JC, et al. Absent right superior vena cava in visceroatrial situs solitus. Am J Cardiol 1997; 80: 175-83.

22) Kawamura I, Hojo R, Fukamizu S. A case of pacemaker implantation in the patient with duplication of the left innominate vein: a case report. Springerplus 2016; 5: 515.

23) Burney K, Young H, Barnard SA, et al. CT appearances of congential and acquired abnormalities of the superior vena cava. Clin Radiol 2007; 62: 837-42.

24) Padhani AR, Hale HL. Mediastinal venous anomalies: potential pitfalls in cancer diagnosis. Br J Radiol 1998; 71: 792-8.

25) Kurihara N, Hirokawa M, Yamamoto T. Postoperative venous thromboembolism in patients undergoing endovenous laser and radiofrequency ablation of the saphenous vein. Ann Vasc Dis 2016; 9: 259-66.

26) Tanaka H, Yamamoto N, Suzuki M, et al. Insufficient lymph drainage causes abnormal lipid accumulation and vein wall degeneration. Ann Vasc Dis 2016; 9: 277-84.

27) Ohmori H, Kanaoka Y, Murata Y, et al. Deep vein thrombosis in patients with severe motor and intellectual disabilities, especially diagnosis and prevention of recurrence for chronic thrombosis - serial changes of sonography and D-dimer. Ann Vasc Dis 2015; 8: 290-6. 